\title{
Microwave Transmission of a Compound Metal Grating
}

\author{
Alastair P. Hibbins, Ian R. Hooper, Matthew J. Lockyear, and J. Roy Sambles \\ Electromagnetic Materials Group, School of Physics, University of Exeter, Exeter EX4 4QL, United Kingdom
}

(Received 6 March 2006; published 27 June 2006)

\begin{abstract}
An array of subwavelength slits in a metallic substrate supports a series of Fabry-Perot-like resonances, where each harmonic results in a transmission peak. Addition of extra slits per period yields a compound grating with a structure factor associated with the basis. In this study each repeat period is comprised of a central slit flanked by a pair of narrower slits. It supports three resonances for every Fabry-Perot-like solution. New and useful insight into this phenomenon is gained by describing each of the modes in terms of the band structure of diffractively coupled surface waves.
\end{abstract}

Over the last few years there has been great interest in the optical properties of metamaterials [1] - objects that owe their electromagnetic behavior as much to their structure as from the materials from which they are composed. Structures studied include arrays of slits, grooves, and holes formed in metals [2-5] and they have been shown to demonstrate remarkable properties such as superdirectivity (beaming) [6-8], enhanced light extraction [9], microscopy [10], angle-independent absorption [11], and "designer" electromagnetic surfaces [12]. Most authors have concluded that many of these phenomena should be attributed to the excitation of surface waves (surface plasmon polaritons, SPPs, at visible frequencies) on the metal $[13,14]$.

Narrow slits or grooves in a metal can support FabryPerot-like modes resulting in a series of resonances in the transmitted and/or reflected signal $[2,4,15,16]$. In the limit of infinitesimal slit width and infinite metal conductivity, these resonant frequencies are given by $\nu_{N}=c N / 2 n t$, where $n$ is the refractive index of the material filling the slits and $t$ is the thickness of the metal substrate. For an array of equally spaced identical cavities there is only one resonance per harmonic $(N)$; however, so-called "phase resonances" are known to break this degeneracy in more complex systems. For example, the excitation of phase resonances has been studied in finite arrays of cavities $[8,17]$. When illuminated with $p$-polarized radiation there are characteristic frequencies at which the phase of the electromagnetic field is established so that its intensity is strongly enhanced inside each cavity. This condition is accompanied by a maximization of the specular efficiency. Similar resonant conditions have also been predicted to exist for infinite "compound" gratings [18,19], where the term "compound" is used to describe a unit cell with a basis (structure factor). The addition of extra cavities to each period introduces new degrees of freedom regarding the possible near-field configurations. The resulting phase resonances are observed as a redistribution of the available energy between propagating orders. For example, the transmission of a compound grating comprised of three narrow slits per period is characterized by a wide peak interrupted by a sharp dip on its low frequency side. Here we provide an experimental study of the microwave transmission of such a compound grating, our experimental measurements confirming the existence of the aforementioned resonances. Importantly, our results also demonstrate coupling to a third resonance away from normal incidence. By recording transmission spectra as a function of the angle of incidence, we show how the character of each of the resonances can be explained in terms of diffractive band folding of surface modes. Our experimental data agree well with numerical model calculations [20].

The experimental sample (Fig. 1) is constructed by stacking together approximately one hundred aluminumalloy slats of thickness $t=19.80 \mathrm{~mm}$, width $w_{m}=$ $3.00 \mathrm{~mm}$, and length $\sim 500 \mathrm{~mm}$, separated from each other by layers of air using small spacers of polyester film at their

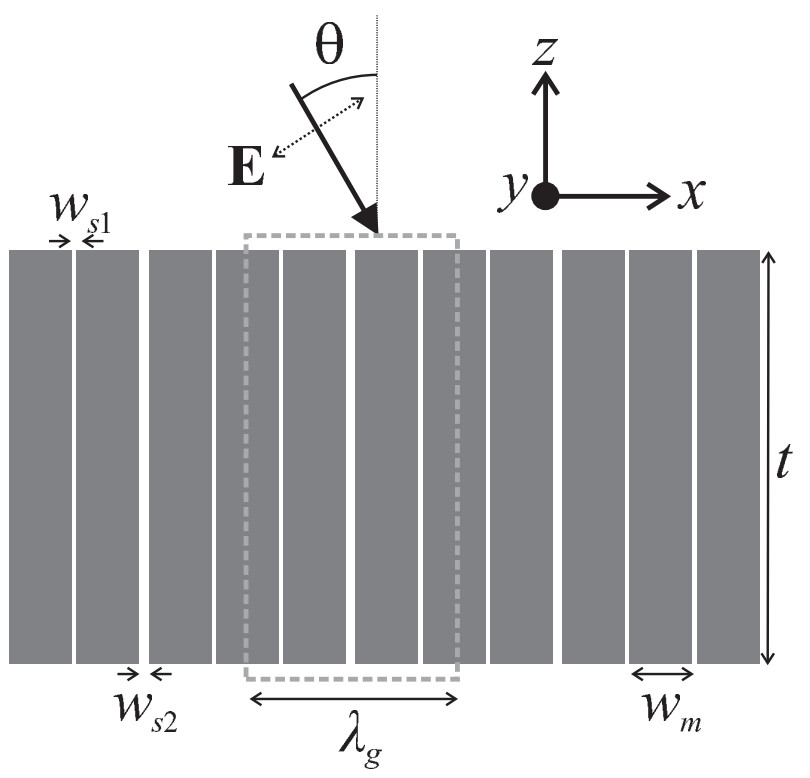

FIG. 1. Sample geometry and coordinate system. 
ends. The three slits forming the basis are not identical: a pair of slits of width $w_{s 1}=0.25 \mathrm{~mm}$ flank a central slit of width $w_{s 2}=0.50 \mathrm{~mm}$, thereby creating a periodicity, $\lambda_{g}=10.00 \mathrm{~mm}$. Microwave radiation is incident upon the sample in the $x z$ plane at an angle $\theta$ from the surface normal and is $p$ polarized. A continuous wave source sweeps the frequency in bands $(12 \leq \nu \leq 18 \mathrm{GHz}$ and $18 \leq \nu \leq 26.5 \mathrm{GHz}$ ) and feeds a fixed position horn antenna. The slats are orientated with their length normal to the plane of incidence, and the sample is positioned on a computer-controlled rotating table to vary $\theta$. In order to obtain averaging of the transmitted signal over a large number of grating periods, the transmitted beam is collected using a spherical mirror before being focused into a second horn antenna (detector).

The normalized experimental transmissivity data at $\theta=$ 0 are shown in Fig. 2 (circles) together with the predictions of the transmissivity and absorption from the numerical model (solid line) [20]. The broad peak in the transmissivity centered at $14.9 \mathrm{GHz}$ and the null at approximately 14.5 GHz correspond to the second harmonic $(N=2)$ Fabry-Perot-like resonance in the slits, whereas the similar features at $22.2 \mathrm{GHz}$ and $21.5 \mathrm{GHz}$ correspond to the third harmonic $(N=3)$. Associated with each of these modes is an increase in absorption in the metal resulting from an intensification of the field strength within the slits. Figures 3(a) and 3(b) show the predicted electric field distribution close to the illuminated surface of the sample at the two resonant frequencies (i.e., at maximum absorption) of the third harmonic modes. It is important to note that for a simply periodic grating with normal incidence illumination the field in each of the slits must be identical. However, with a compound grating new degrees of freedom are introduced. The structure under study here supports two possible phase configurations at $\theta=0$. These correspond to an identical phase of the field in each of the

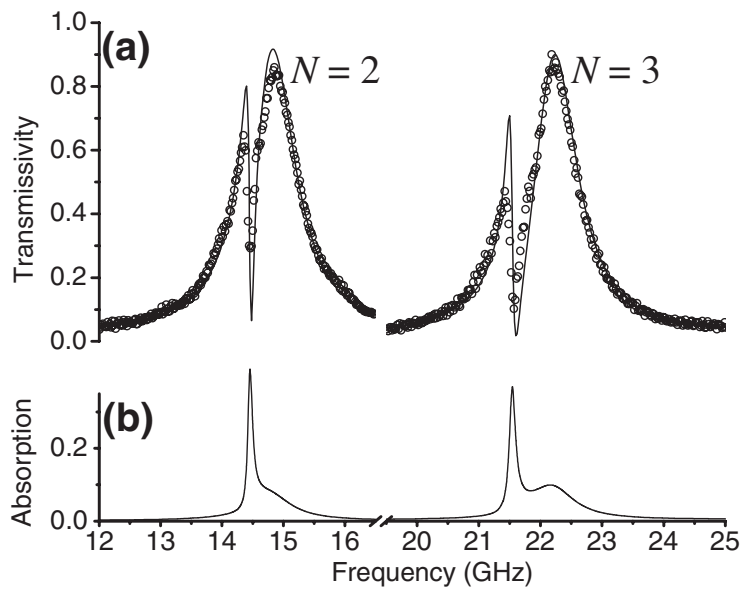

FIG. 2. (a) Experimental transmissivity spectrum $(\bigcirc)$ from the sample illuminated at normal incidence $(\theta=0)$. The solid line represents the predictions from the numerical model. (b) Predicted absorption spectrum. slits (" +++ "), and the other when the field in the central slit is completely out of phase with that in the two narrower slits [" -+- ", or the " $\pi$ " resonance [17] ]. It is evident from Fig. 3 that the broad peaks are the primary resonance with the incident field driving all three slits in phase (" +++ "). The much narrower feature in the low frequency wing of each broad peak is the $\pi$ resonance, but direct coupling to the mode is not possible since the electric field in the narrow slits is out of phase with the incident field. Therefore, observation of the $\pi$ resonance can only be expected in the wings of the primary resonance since the mode can only be excited via coupling between the slits. The transmission associated with each of these two modes interferes resulting in a Fano-shaped feature in the recorded spectra. The predicted phase difference of the electric field between the central and adjacent slits is also plotted in Fig. 3 as a function of frequency $(\Delta \phi$, calculated at the illuminated surface at the midpoints of the slit widths). At frequencies below the resonant conditions the phase difference is zero. As the frequency is incremented there is a phase change of order $\pi$ radians resulting in the
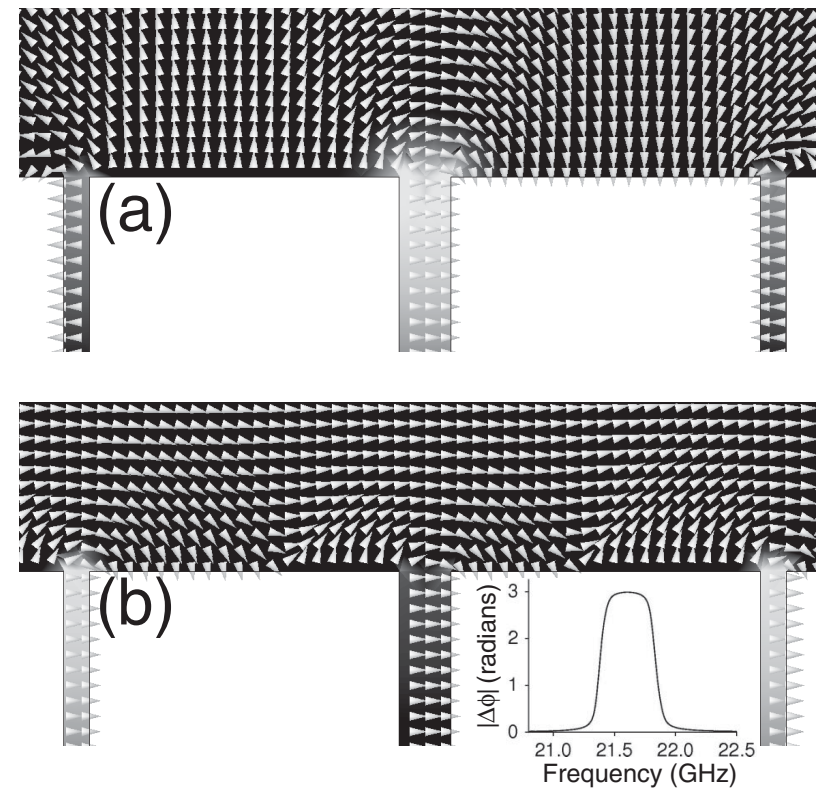

FIG. 3. Predictions of the electric field distribution in the $x z$ plane on resonance of the two $N=3$ modes with normal incidence illumination. For clarity only part of the unit cell is shown (approximately $4 \mathrm{~mm}$ either side of the central slit, and $\sim 1.5 \mathrm{~mm}$ above and below the upper surface). The gray scale illustrates time-averaged electric field magnitude and the arrowheads indicate the direction of the electric field vector at a phase in its temporal cycle corresponding to maximal intensification. (a) The $\pi$ mode " -+- " at $21.5 \mathrm{GHz}$ and (b) the primary mode " +++ " at $22.2 \mathrm{GHz}$. The lightest shading corresponds to field enhancement of at least 30 and 15 times, respectively. The inset in (b) illustrates the difference in phase of the electric field as a function of frequency at the illuminated surface between points located at the midwidth of the central and adjacent slit. 
" -+- " configuration on passing through the resonance of the $\pi$ mode.

By placing the sample on a computer-controlled rotating table, we directly record and plot in Fig. 4 the photonic band structure of the sample as a function of the in-plane wave vector $\left[k_{x}=(2 \pi \nu / c) \sin \theta\right]$ at frequencies close to the third Fabry-Perot-like harmonic. The primary transmission peak No. 3 is flat banded [2,4]; however, more surprising is the curvature of the lowest energy band No. 1 , corresponding to the $\pi$ resonance (" -+- ") at normal incidence. Its dispersion seemingly resembles that of a grating-coupled SPP whose propagation has been strongly perturbed by the grating profile $[4,11,21]$. This surfacewave-like characteristic is verified by plotting the numerically modeled Poynting vector distribution at resonance for $\sin \theta=0.3$ (Fig. 5). Evidently this mode should not be attributed to a single surface wave, but to a pair of identical SPPs on the upper and lower surfaces of the array coupled together via the Fabry-Perot-like waveguide resonances supported by the slits.

On close inspection of the experimental transmissivities (Fig. 4), a third feature positioned between the two aforementioned modes can be seen (mode No. 2), and it is associated with the phase shift that returns the field configuration to " +++ " (Fig. 3 inset). The mode occurs at a frequency at which $\Delta \phi=\pi / 2$ corresponding to a $\pi$-radian phase difference between the resonant fields of the two narrow slits, accompanied by an absence of coupling to the mode in the central slit ("+0-"). Clearly, excitation of this asymmetric resonance is impossible at normal incidence, and the coupling strength clearly lessens as the angle is reduced (Fig. 4). Since the horn antennae do not transmit a perfectly planar beam, we also observe this resonance as a very weak feature in the normal incidence

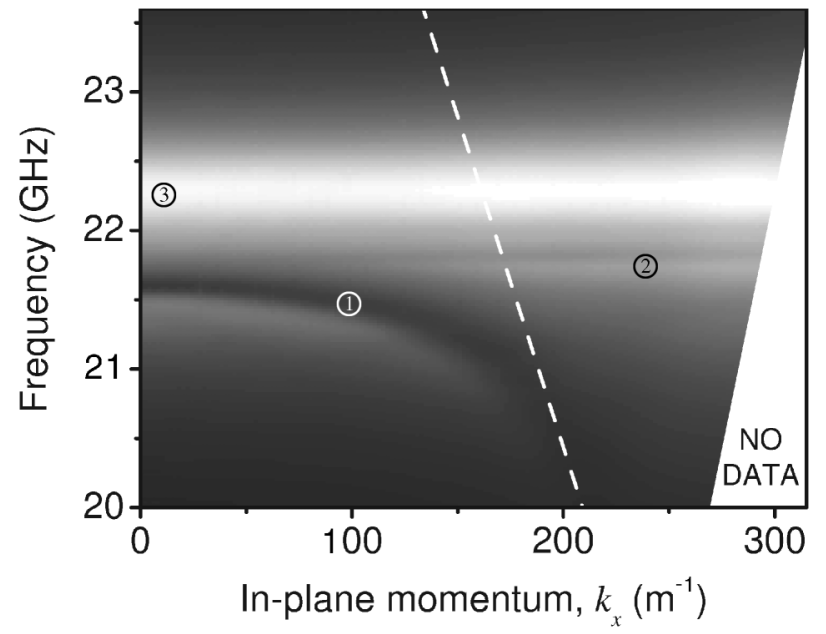

FIG. 4. Experimental transmissivity spectra plotted as a function of $k_{x}=(2 \pi \nu / c) \sin \theta$. White regions correspond to normalized transmissivities in excess of $90 \%$. The white dashed line corresponds to the first order diffracted light line (scattered by a single grating wave vector, $k_{g}$ ). experimental data for each of the harmonics (at $\sim 14.6 \mathrm{GHz}$ and $\sim 21.8 \mathrm{GHz}$ ).

The photonic band structure of SPPs on simple, shallow metallic gratings is well understood [22], whereas the current structure represents a very deep grating with compound (multiperiodic) structure. However, it is not too dissimilar to a reflection grating previously studied by the present authors [11] and one may use similar reasoning here to discuss our current results. One may effectively reduce the analysis of the band structure to just two components of the grating profile: a very large amplitude, short pitch component (of period $\lambda_{g} / 3$, i.e., the closely spaced deep slits) convolved with a much weaker longer pitch component $\left(\lambda_{g}\right.$, associated with the different width of every third slit). Scattering of the modes by the short pitch component, $3 k_{g}$ (where $k_{g}=2 \pi / \lambda_{g}$ ), creates huge gaps in the SPP band structure at the corresponding Brillouin zone boundaries $\left(k_{x}=0\right.$ and $\left.3 k_{g} / 2\right)$. The perturbation of the profile from a flat surface is so great that the fields associated with the surface mode couple together across the narrow slit and become highly localized (self-coupled SPPs) $[4,21]$. In the limit of perfect conductivity and subwavelength slit width these cavity modes are entirely equivalent to the Fabry-Perot-like standing wave resonances of the TEM (transverse electromagnetic) waveguide mode (order $N$ ). Whilst the lowest energy of these bands exists outside the incident light line, higher order $(N \geq 1)$ diffractively coupled modes are observed inside the radiative region. These are the origin of the broad transmission peaks in Fig. 2, i.e., the mode associated with the " +++ " phase configuration and band No. 3 in Fig. 4. An illustration of a similar, highly perturbed band structure can be found in Ref. [21]. Note that although the structure studied there is a silver reflection grating, comparison of the band structure to that of the present study is valid for frequencies well below the surface plasma frequency $\left(\omega_{p} / \sqrt{2}\right)$, for which the nonperturbed SPP dispersion closely follows the light line. The much narrower features (i.e., the $\pi$ mode at normal incidence, and the two bands labeled No. 1 and No. 2 in Fig. 4) are a consequence of convolving the short pitch component of the profile with the long-pitch component. While the longpitch component is too weak to cause any significant perturbation of the shape of the SPP dispersion curve, it will introduce important band-folding effects. Sections of the dispersion curve that exist outside the incident light line on a simple grating are folded into the radiative region [see Fig. 3 of Ref. [11]]. Hence the originally nonradiative modes associated with the low energy edge of the band gap at $k_{x}=3 k_{g} / 2$ become excitable through scattering by integer multiples of $k_{g}$. These resonances are therefore narrower than the strongly diffracted primary mode. Furthermore, it is clear that they must always occur at a lower frequency than the primary mode since they correspond to modes of a lower energy band edge [4]. Band 

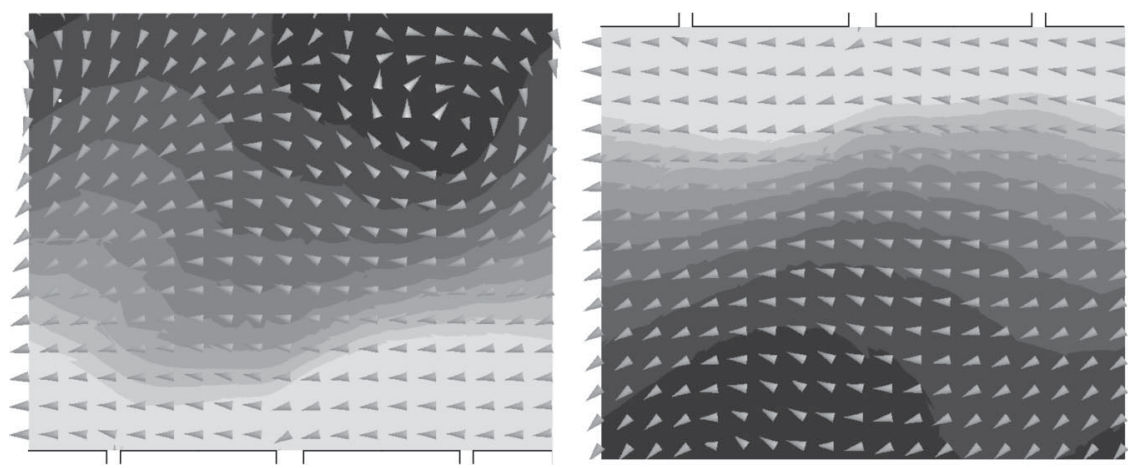

FIG. 5. Predictions of the Poynting vector in the $x z$ plane in regions approximately $10 \mathrm{~mm}$ above (left) and below (right) the upper and lower surfaces of the sample, respectively. The gray scale represents the magnitude and the arrowheads show the direction of the vector on resonance of the coupled surface-wave associated with the $N=3$ harmonic with $\sin \theta=0.3$ (band No. 1 at $\nu=21.2 \mathrm{GHz}$, see Fig. 4). Regions of lightest shading correspond to power enhancements of at least 11 times compared to the incident field.

No. 1 results from band folding of the curved part of the dispersion into the radiative region (i.e., scattering from $\pm k_{g}= \pm 2 \pi / \lambda_{g}$ ), whereas the band labeled No. 2 is a consequence of scattering of the more flat-banded parts (i.e., scattering via $\pm 2 k_{g}$ ). This SPP-band-folding picture verifies the periodicity of the phases of the field at each resonance. It is interesting to note that the order of the two lowest energy modes can be reversed by changing the profile. For example, a basis formed of a narrow central slit flanked by two wider slits shows a similar response to that seen here at normal incidence. However, on increment of the angle it is clear that the mode associated with the $\pi$ resonance at normal incidence remains flat banded, whereas the asymmetric mode, which appears at a slightly lower frequency, disperses in a SPP-like manner [11].

In this work we have experimentally studied and numerically modeled the transmissivity of an air-filled compound grating comprised of two narrow slits flanking a wider central slit. In contrast to a simply periodic structure, we have shown it to support not one, but two cavity resonances per Fabry-Perot-like harmonic at normal incidence. In addition, by studying the transmissivity spectra as a function of the angle of incidence we observe a third resonance that can only be coupled to if a phase variation exists across the surface. We identify and describe each of the three modes in terms of the band structure of diffractively coupled surface waves (SPPs) that propagate on each surface of the sample, and whose fields interact via the Fabry-Perot-like modes within the slits. At normal incidence the standing wave states of these surface waves correspond to three unique phase configurations.

The authors would like to acknowledge the financial support of the Engineering and Physical Sciences Research Council (EPSRC, UK).
[1] D. R. Smith, J. B. Pendry, and M. C. K. Wiltshire, Science 305, 788 (2004).

[2] J. A. Porto, F. J. García-Vidal, and J. B. Pendry, Phys. Rev. Lett. 83, 2845 (1999).

[3] J. R. Andrewartha, J. R. Fox, and I. J. Wilson, Opt. Acta 26, 197 (1979).

[4] W.-C. Tan et al., Phys. Rev. B 59, 12661 (1999).

[5] T. W. Ebbesen and H. J. Lezec, Nature (London) 391, 667 (1998).

[6] H. J. Lezec et al., Science 297, 820 (2002).

[7] A.P. Hibbins, J. R. Sambles, and C. R. Lawrence, Appl. Phys. Lett. 81, 4661 (2002).

[8] D.C. Skigin and V. V. Veremey, IEEE Trans. Antennas Propag. 47, 376 (1999).

[9] C. Liu, V. Kamaev, and Z. V. Vardeny, Appl. Phys. Lett. 86, 143501 (2005).

[10] Y. Garini et al., Proc. SPIE 5327, 115 (2004).

[11] A. P. Hibbins, J. R. Sambles, and C. R. Lawrence, Appl. Phys. Lett. 80, 2410 (2002).

[12] A. P. Hibbins, B. R. Evans, and J. R. Sambles, Science 308, 670 (2005).

[13] A. Krishnan et al., Opt. Commun. 200, 1 (2001).

[14] W. L. Barnes, A. Dereux, and T.W. Ebbesen, Nature (London) 424, 824 (2003).

[15] S. Astilean, Ph. Lalanne, and M. Palamaru, Opt. Commun. 175, 265 (2000).

[16] H. E. Went et al., Appl. Phys. Lett. 77, 2789 (2000).

[17] V. V. Vladimir, IEEE. Trans. Antennas Propag. 46, 494 (1998).

[18] A. N. Fantino, S. I. Grosz, and D. C. Skigin, Phys. Rev. E 64, 016605 (2001).

[19] D. C. Skigin and R. A. Depine, Phys. Rev. Lett. 95, 217402 (2005).

[20] Finite element method computer modeling was done with the use of HFSS (Ansoft Corporation, Pittsburgh, PA).

[21] I. R. Hooper and J. R. Sambles, Phys. Rev. B 65, 165432 (2002).

[22] W. L. Barnes et al., Phys. Rev. B 54, 6227 (1996). 